\title{
Deciphering the Molecular Landscape of Appendiceal Mucinous Neoplasms for Diagnostic, Prognostic and Therapeutic Purposes
}

\author{
Haroon A. Choudry, MD, FACS, and David L. Bartlett, MD \\ Division of Surgical Oncology, Department of Surgery, University of Pittsburgh Medical Center, Pittsburgh, PA
}

I was delighted to read the latest effort by Dr. Levine's group, at Wake Forest Cancer Center, to identify a prognostically relevant gene expression-based tumor subtyping classification system for appendiceal mucinous neoplasms $(\mathrm{AMN})$. While we have made progress in developing clinically relevant guidelines for classifying these rare tumors using standard cytohistopathologic features [e.g., consensus classification proposed by the Peritoneal Surface Oncology Group International (PSOGI) in 2016; and the updated 8th edition of the American Joint Committee on Cancer (AJCC) in 2017], there continues to be significant variability and unpredictability in biological behavior, oncological outcomes, and treatment-response within and across the histologically defined subgroups of AMN following standard therapies. ${ }^{1,2}$ A comprehensive understanding of the molecular landscape of AMN would help stratify patients into biologically homogeneous tumor subtypes, with more predictable biological behavior and response to drug therapy.

As opposed to genetic mutational analysis, transcriptome-level data provides a comprehensive picture of altered molecular signaling pathways and are closely linked to clinical phenotype, biological behavior, and response to chemotherapeutic/biological drugs. Gene expression-based analyses have been performed for a variety of malignancies, including breast and colorectal cancers, leading to the identification of discrete molecular subtypes within these cancers. ${ }^{3-7}$ In this vein, Dr. Levine's group utilized a custom-designed NanoString 148-gene

\footnotetext{
(C) Society of Surgical Oncology 2020
}

First Received: 21 December 2019;

Published Online: 23 January 2020

H. A. Choudry, MD, FACS

e-mail: choudrymh@upmc.edu panel for gene-expression profiling of low- and high-grade AMN (low-grade: 76 cases; high-grade: 38 cases), pathologically classified according to the two-tiered Bradley classification system. ${ }^{8}$ A significant number of cases (44\%) were initially excluded from analysis due to low cellularity $(n=51)$, suboptimal RNA quality $(n=37)$, or unreliable gene expression profiles $(n=20)$. Utilizing various statistical techniques, including signed non-negative matrix factorization (sNMF), topology learning, reverse graph embedding and cross-cohort performance analysis, three molecular subtypes were discerned by the altered expression patterns of 17 genes. Subtypes were termed: (1) immune-enriched (IE, $n=40$ ), expressing immune-cell related genes and demonstrating favorable prognosis (3year OS/PFS 83\%/65.5\%); (2) oncogene-enriched (OE, $n=43$ ), expressing genes associated with cancer growth/ progression and demonstrating poor prognosis (3-year OS/ PFS 36\%/14\%); and (3) mixed (M, $n=55)$, with overlapping gene expression and demonstrating intermediate prognosis (3-year OS/PFS 55\%/30\%). Moreover, the 17-gene signature-based 3-tiered molecular subtypes were confirmed in an independent set of 40 patients previously profiled by the same team on the Affymetrix U133A GeneChip platform. Finally, in a multivariable Cox-regression analysis, the identified molecular subtypes were prognostic for survival (independent of pathologic grade and completeness of cytoreduction). This study identified prognostically relevant molecular signature-based subtypes within AMN, highlighted a potential role for the immune system in these tumors, and elucidated potential molecular targets/pathways to help refine therapeutic strategies for these rare tumors.

While this study is an important step towards deciphering the molecular landscape of AMN, experience from similar research in other cancers highlights a broad range of issues that need to be considered before gene 
expression-based molecular subtyping can have a significant impact on clinical practice. Differences in RNA extraction methods, gene expression profiling platforms, and data analysis pipelines are known to influence subtype affiliation for individual cancer patients. Similarly, tumor subtype affiliation can be influenced by intra-tumoral heterogeneity (i.e., tumor subtyping can vary in different regions or even cells within the same tumor), variability in hard-wired signaling pathways native to the hierarchical cell-of-origin being transformed (i.e., different signaling pathways may be activated in stem cells versus differentiated cells during transformation), tumor stage at the time of analysis (i.e., gene expression profiles may vary in early versus late stage, or primary versus recurrent, or chemotherapy-naïve versus chemo-experienced, or local versus metastatic tumors), underlying genetic mutations and sequence of mutations, and external pressures within the tumor microenvironment (i.e., stromal cells or prevailing microbiome). In colorectal cancer, inconsistencies related to the above-mentioned biological complexities have hampered the reproducibility and clinical applicability of gene expression-based tumor subtyping classification systems. The existence of such complexity within and across tumors can be daunting and challenging; however, such molecular diversity provides an opportunity for personalized cancer medicine tailored to the unique attributes of a specific tumor subtype.

Ultimately, it is most likely that a combination of features (histopathologic, chromosomal alterations, genetic mutations, gene expression profiles, proteomic, and posttranslational regulatory changes) from both the tumor and the microenvironment will best define the molecular landscape of individual cancers. Deciphering the molecular landscape of AMN will enable uniform categorization of biologically homogeneous tumor subtypes (genetic signatures) and allow for subtype-specific treatment regimens.

DISCLOSURE None.

\section{REFERENCES}

1. Carr NJ, Cecil TD, Mohamed F, Sobin LH, Sugarbaker PH, Gonzalez-Moreno S, et al. A consensus for classification and pathologic reporting of pseudomyxoma peritonei and associated appendiceal neoplasia: the results of the Peritoneal Surface Oncology Group International (PSOGI) Modified Delphi Process. Am J Surg Pathol. 2016;40(1):14-26.

2. Choudry HA, Pai RK. Management of mucinous appendiceal tumors. Ann Surg Oncol. 2018;25(8):2135-44.

3. Fessler E, Medema JP. Colorectal cancer subtypes: developmental origin and microenvironmental regulation. Trends Cancer. 2016;2(9):505-18.

4. Guinney J, Dienstmann R, Wang X, de Reynies A, Schlicker A, Soneson $\mathrm{C}$, et al. The consensus molecular subtypes of colorectal cancer. Nat Med. 2015;21(11):1350-6.

5. Sadanandam A, Lyssiotis CA, Homicsko K, Collisson EA, Gibb WJ, Wullschleger $\mathrm{S}$, et al. A colorectal cancer classification system that associates cellular phenotype and responses to therapy. Nat Med. 2013;19(5):619-25.

6. Perou CM, Sorlie T, Eisen MB, van de Rijn M, Jeffrey SS, Rees CA, et al. Molecular portraits of human breast tumours. Nature. 2000;406(6797):747-52.

7. 7. Russnes HG, Lingjaerde OC, Borresen-Dale AL, Caldas C. Breast cancer molecular stratification: from intrinsic subtypes to integrative clusters. Am J Pathol. 2017;187(10):2152-62.

8. Su J, Jin G, Votanopoulos K, Craddock L, Shen P, Chou J, et al. Prognostic molecular classification of appendiceal mucinous neoplasms treated with cytoreductive surgery and hyperthermic intraperitoneal chemotherapy. Ann Surg Oncol. 2020. https://doi. org/10.1245/s10434-020-08210-5.

Publisher's Note Springer Nature remains neutral with regard to jurisdictional claims in published maps and institutional affiliations. 